\title{
Medium- and long-term effects of endovascular treatments for severely stenotic basilar arteries supported by multimodal imaging
}

Yuanzhi $\mathrm{Li}^{1 \dagger}$, Zhenfa $\mathrm{Li}^{2^{*}}$, Ligang Song ${ }^{3^{*}}$, Weimin Xie ${ }^{4 \dagger}$, Xianghao Gong ${ }^{5}$, Dongliang $\mathrm{He}^{6}$ and Xin Zhang ${ }^{7}$

\begin{abstract}
Background: To evaluate the medium-and long-term effect of intravascular interventional therapy for symptomatic severe basilar artery stenosis supported by multimodal imaging.

Method: After strict screening of 67 patients with symptomatic severe basilar artery stenosis (70-99\%) with atherosclerotic stenosis, 67 patients with symptomatic recurrence after intensive drug treatment were treated with intravascular balloon dilatation and Enterprise stent implantation. Any stroke or death within 30 days after operation and any stroke and restenosis during medium-and long-term follow-up were recorded.

Results: (1)The mean age of 67 patients (67lesions) was $57 \pm 8$ years old, and the technical success rate was 100\%; (2) Preoperative angiography showed that the collateral circulation was poor, and $\mathrm{TICI}$ was $1-2 \mathrm{a}$ while postoperative angiography showed that $\mathrm{TICl}$ was significantly improved to 2b-3; (3)The average preoperative stenosis rate was $82 \pm 9 \%$, and the postoperative stenosis rate was reduced to $17 \pm 10 \%$; (4) Before surgery, abnormal perfusion was found in the posterior circulation CTP; After the postoperative re-examination, the posterior circulation of CTP perfusion was significantly improved; (5) Postoperative symptoms and neurological conditions improved significantly; (6) Complications of perforating branch event occurred in 1 case after operation, and symptoms were relieved after more than 1 month of medication treatment, and mild neurological dysfunction remained. 1 case developed subacute thrombosis in the stent, which improved after active intra-arterial thrombolysis, and there was no residual neurological dysfunction; and 1 case of micro-guide wire being trapped by the distal vasospasm. (7)67 patients were followed up by telephone, WeChat or imaging for 36-66 months.

(Continued on next page)
\end{abstract}

\footnotetext{
*Correspondence: lizhenfa8770@163.com; bjsongligang@163.com

${ }^{+}$Yuanzhi Li is the first co-first author and Weimin Xie is the second co-first author.

${ }^{2}$ Department of Vascular Surgery, Affiliated Hengyang Hospital, Southern

Medical University (Hengyang Central Hospital), 12\# Yancheng road,

Hengyang 421001, Hunan province, China

${ }^{3}$ Department of Neurointervention, Affiliated Tiantan Hospital, Capital

Medical University, 119\# west south fourth ring road, fengtai district, Beijing

100050, China

Full list of author information is available at the end of the article
}

(c) The Author(s). 2020 Open Access This article is licensed under a Creative Commons Attribution 4.0 International License, which permits use, sharing, adaptation, distribution and reproduction in any medium or format, as long as you give appropriate credit to the original author(s) and the source, provide a link to the Creative Commons licence, and indicate if changes were made. The images or other third party material in this article are included in the article's Creative Commons licence, unless indicated otherwise in a credit line to the material. If material is not included in the article's Creative Commons licence and your intended use is not permitted by statutory regulation or exceeds the permitted use, you will need to obtain permission directly from the copyright holder. To view a copy of this licence, visit http://creativecommons.org/licenses/by/4.0/. The Creative Commons Public Domain Dedication waiver (http://creativecommons.org/publicdomain/zero/1.0/) applies to the data made available in this article, unless otherwise stated in a credit line to the data. 
(Continued from previous page)

Conclusions: In summary intravascular balloon dilation + Enterprise stent implantation is safe and effective for the treatment of symptomatic severe atherosclerotic stenosis of the basilar artery, with high technical success rate, low perioperative complications, and good mid-term and long-term effects.

Keywords: Basilar artery, Stenosis, Atherosclerosis, Stent implantation, Angioplasty

\section{Background}

Stroke is one of the leading causes of disability and death in adults. Intracranial arterial stenosis is the narrowing of the main intracranial arteries due to the formation of atherosclerotic plaques, which may represent the most common cause of stroke worldwide. Intracranial arterial stenosis is more common in Asians, particularly in the Chinese population. Among all stroke populations in China, The incidence and prevalence of ischemic stroke are as high as 69.6 and $77.8 \%$, respectively [1], and have been increasing annually and are more commonly observed in younger populations [2]. More than $40 \%$ of ischemic stroke cases are related to compromised blood circulation of the vertebrobasilar artery [3]. According to the Chinese Intracranial Atherosclerosis (CICAS) study [4], the prevalence of intracranial atherosclerotic stenosis (ICAS) is as high as $46.6 \%$ in the Chinese population of stroke patients, and the basilar artery is the most vulnerable component of the affected blood vessels. Even following adherence to current standard medications, patients still exhibit high morbidity. The risk of disease-related ischemic stroke is especially high in symptomatic patients with a high degree of atherosclerotic stenosis (>70\%) [5]. Therefore, development of effective interventions to prevent stroke in patients with ICAS is necessary, especially for those with symptomatic ICAS. To this aim, leveraging technological advancements and updated materials may facilitate development of safer and more efficacious endovascular stent implantations for the treatment of symptomatic severe basilar artery stenosis. The present study screened 67 patients with symptomatic atherosclerotic stenosis in the basilar arteries and evaluated the safety, as well as the medium- and long-term effects, of endovascular interventions with an Enterprise stent system.

\section{Methods}

\section{Research subjects}

Sixty-seven patients with severe atherosclerotic stenosis in the basilar arteries who were admitted and treated with Enterprise stents at Tiantan hospital, Hengyang central hospital and Zhujiang hospital from April 2014 to April 2016 were continuously screened. All patients signed written informed consents before participating in the study, and the research protocol of this study was approved by the Ethics committee of the hospital. Inclusion criteria for patients in the present study were as follows: (1) 18-80 years old; (2) 70-99\% extent of stenosis with the length of stenosis being less than $15 \mathrm{~mm}$, as measured based on the standard vertebral method established by the Warfarin-Aspirin Symptomatic Intracranial Disease (WASID) trial [6], using the same image magnification in digital subtraction angiography (DSA); (3) standard medical drug treatment for 1-3 months and non-disabling posterior circulation infarct $(\mathrm{PCI})$ and transient ischemic attack (TIA) recurrence in the area receiving blood supply from the affected vessels; and (4) the last episode of symptoms occurring at least 3 weeks before the scheduled operation. Exclusion criteria for patients in the present study were as follows: (1) presence of non-atherosclerotic stenosis; (2) symptoms of perforating stroke; (3) potential cardiac-derived embolism, intracranial hemorrhage within 3 months, intracranial tumors, aneurysms, venous malformation, or moyamoya disease; or (4) abnormal blood coagulation, clopidogrel resistance, or presence of heparin, aspirin, or clopidogrel contraindications.

\section{Preoperative assessments}

All patients were preoperatively assessed by multimodal imaging, including transcranial Doppler sonography (TCD), computed tomography angiography (CTA), computed tomography perfusion (CTP), magnetic resonance angiography (MRA), diffusion weighted imaging (DWI), high-resolution magnetic resonance imaging (HR-MRI), perfusion-weighted imaging (PWI), and digital subtraction angiography (DSA) of the entire cerebrovascular system. Measurement of stenosis was performed according to the standard vertebral method established by the WASID. The DSA images at the same magnification were used to measure the degree of stenosis, the length of stenosis, the conditions of proximal and distal lesions, the relationship between branch vessels and plaques, and the collateral circulation and forward blood flow conditions of the patients. CTP or PWI was used to evaluate cerebral perfusion; HR-MRI was used to determine the eccentricity and concentricity of the plaque.

\section{Surgical procedures}

All operations in this study were performed under general anesthesia. Systemic heparinization was induced in 
all patients throughout the surgical procedures. A $6 \mathrm{~F}$ or 5F Envoy guiding catheter (Cordis, Santa Clara, CA) was placed into the distal segment of the V2 vertebral artery at a level of approximately $\mathrm{C} 4-\mathrm{C} 2$ of the cervical spine (depending on the diameter of the target vertebral artery). A transradial approach was used instead of a transfemoral approach if there was difficulty in reaching the target vessel. A dosage of $70 \mathrm{U}$ of heparin $/ \mathrm{kg}$ bodyweight was injected after the completion of intubation. The degree of stenosis was calculated based on the diameter of the normal vessel (WASID standard) near the distal end of the lesion, and was either calibrated with a contrastfilled catheter as a reference or was measured by a DSA workstation. All lesions were pre-expanded with a Gateway balloon (Boston, MA), followed by implantation with an Enterprise stent (Cordis). According to our experience, the selection of the Gateway balloon was comprehensively based on the distribution of the adjacent perforating branch of the lesion, the degree of stenosis, the length of the lesion, the degree of angulation of the lesion, the location and texture of the plague, and 70$80 \%$ of the normal blood vessel diameter at the distal end of the lesion. In addition, multiple expansions of the balloon were avoided. Patients with preoperative assessments of hard plaque textures were subjected to extended balloon expansions lasting 3 min under the corresponding pressure until achieving sub-satisfactory results of the lesion angioplasty. Stent selection was based on both ends of the stent exceeding the lesion by $3-5 \mathrm{~mm}$. Although the Enterprise stent could be recycled and released repeatedly, every effort was made for its accurate placement in the first release to avoid multiple releases and cutting of the plaque. After the stent release was completed, a vertebral arteriography was performed immediately, and the residual stenosis was measured. The stenosis was observed simultaneously for $10 \mathrm{~min}$, and an angiography was performed to determine the forward blood flow. In addition to the intraoperative heparinization, nimodipine was used to prevent cerebral vasospasms and to control systolic blood pressure to reside between 110 and $130 \mathrm{mmHg}$. The criteria for successful stent implantation consisted of complete coverage of the lesion by the stent, no drift of the stent, no new neurological deficits, and $<50 \%$ residual stenosis (Fig. 1).

\section{Perioperative management}

All patients were subjected to risk-factor control and improvement of living habits. Patients were also orally administered aspirin $(100 \mathrm{mg} /$ day $)$ and clopidogrel $(75 \mathrm{mg} /$ day) for more than five consecutive days before the operation. Patients who had poor thromboelastographies
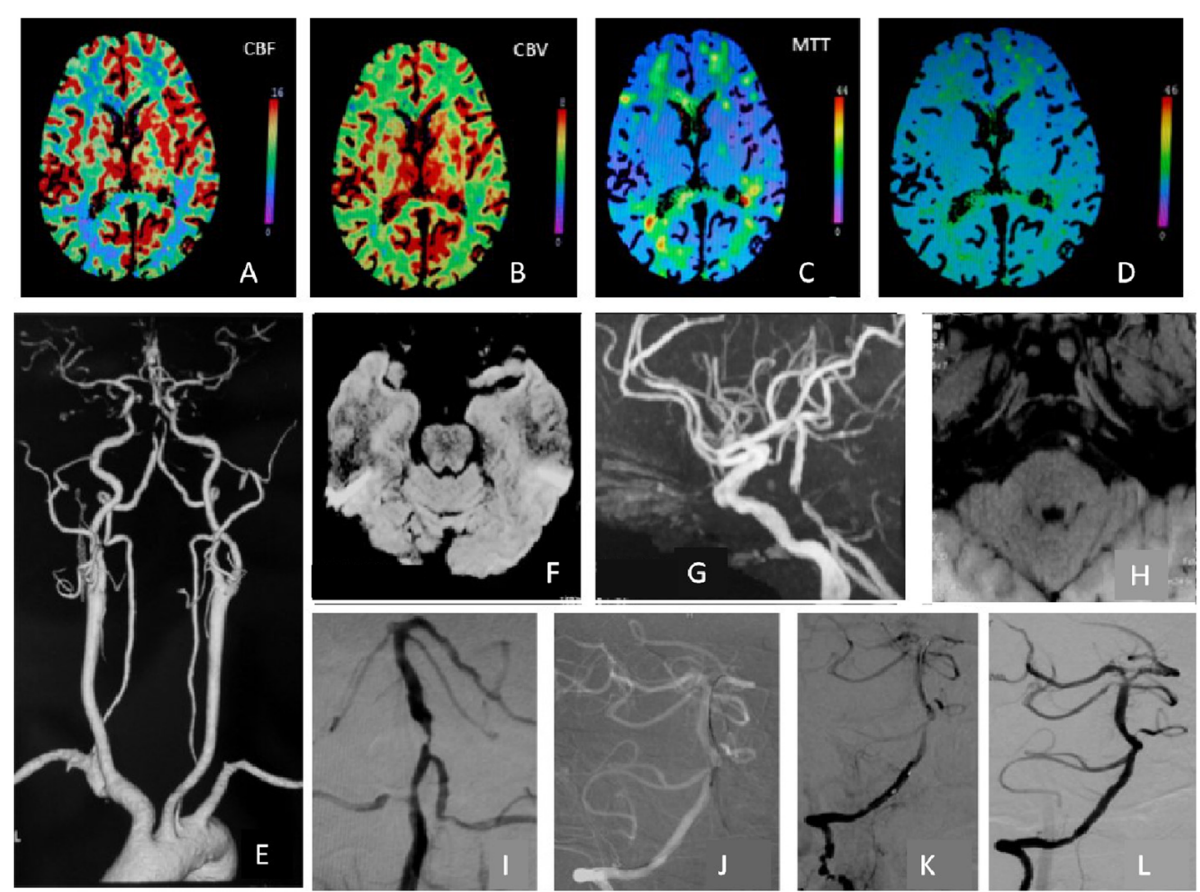

Fig. 1 Multimodal imaging assessment and surgical procedures. a-d Preoperative, posterior circulation CTP perfusion was abnormal. e CTA of the head and neck demonstrates severe stenosis of the lower basilar artery. (-h DWI images showed no acute cerebral infarction, MRA images show severe stenosis in the lower basilar artery, HR-MRI images show basilar eccentric stenosis. i Before the operation, DSA images showed severe stenosis in the lower basilar artery. j-k During the procedure, balloon expansion and stent implantation were performed. I Postoperative, DSA images showed significant improvement in severe basilar stenosis 
after antiplatelet aggregation medication were subsequently treated with antiplatelet drugs.

Intravenous nimodipine $(4.2 \mathrm{ml} / \mathrm{h})$ was given continuously for $2 \mathrm{~h}$ before the operation to prevent cerebral vasospasms. A complete skull CT immediately after surgery was used to confirm no intracranial hemorrhage. Each patient was given moderate sedation in the neurological intensive care unit and a subcutaneous injection of low-molecular-weight heparin once every $12 \mathrm{~h}$ for two to 3 days. After the stent implantation, each patient was given $100 \mathrm{mg} /$ day of aspirin and $75 \mathrm{mg} /$ day of clopidogrel for 90 days, followed by continued administration of $100 \mathrm{mg} /$ day of aspirin for life. For postoperative riskfactor control, systolic blood pressure and low-density lipoproteins of the patients were controlled under 130 $\mathrm{mmHg}(<120 \mathrm{mmHg}$ for diabetic patients) and $70 \mathrm{mg} / \mathrm{dl}$ $(1.81 \mathrm{mmol} / \mathrm{l})$, respectively, or low-density lipoproteins were reduced by $50 \%$. Patients were asked to quit smoking, change their eating habits, lose weight, avoid being sedentary, and exercise properly.

\section{Follow-ups}

Patients were postoperatively followed up by telephone, WeChat, or imaging review. The assessments were performed by the same neurologist who performed the surgery, with PCI, TIA, and incidence of death being monitored for 30 days. The National Institute of Health stroke scale (NIHSS) was used for assessments of patients before admission and at discharge. During the clinical follow-ups, patients who were suspected to have recurrent stroke were subjected to head CT or MRI examinations. Patients with stent implantations were followed up 3 months after being discharged, and their TCD/CTA, MRA, or DSA were reviewed after 6-12 months. Patients who were asymptomatic were followed up every 6 months. The success rate of stent implantation, incidence of perioperative complications, degree and rate of restenosis, speed of stenotic blood flow, and incidence of ischemic events of all patients were recorded. Restenosis was considered as the post-stent implantation arterial diameter being less than $50 \%$ of the immediate arterial diameter after the implantation.

\section{Statistical analysis}

All statistical analysis was performed using SPSS 22.0 software (IBM SPSS Inc., Chicago, IL). Data are presented as the mean \pm standard deviation $(x \pm s)$. Comparisons between two distributions were performed using $t$-tests or Wilcoxon. Comparisons among more than two distributions were performed by one-way analyses of variance (ANOVAs). Categorical counted data were compared using chi-square $\left(\chi^{2}\right)$ tests. A $P<0.05$ was considered statistically significant.

\section{Results}

Results after stent implantations

A total of 67 patients ( $57 \pm 8$ years old), including 57 males and 10 females, were included in the present study. Symptoms lasted from 3 weeks to 90 days after the stent implantations. A total of 67 Enterprise stents were implanted into the patients, with a $100 \%$ technical success rate. The average preoperative stenosis rate was $82 \pm 9 \%$, and the postoperative stenosis rate was reduced to $17 \pm 10 \%$. There were 38 cases of $\leq 5$ - $\mathrm{cm}$ lesion lengths, 18 cases of 5-10-cm lesion lengths, and 11 cases of 11-15-cm lesion lengths. The average stenosis length was $7 \pm 4 \mathrm{~mm}$. Before the stent implantation, there were eight cases of TICI grade 1 (11.9\%), 47 cases of TICI grade $2 \mathrm{a}(70.2 \%)$, and 12 cases of TICI grade $2 \mathrm{~b}(17.9 \%)$; after the stent implantation, there were 37 cases of TICI grade $2 \mathrm{~b}(55.2 \%)$ and 30 cases of TICI grade 3 (44.8\%) (Table 1). Comparison of pre- and postoperative results revealed a significant improvement of forward blood flow $\left(X^{2}=97.755, P<0.001\right)$. The peak blood flow velocity in the basilar artery stenosis monitored by TCD was significantly decreased from $226 \pm 21 \mathrm{~cm} / \mathrm{s}$ preoperatively to $127 \pm 13 \mathrm{~cm} / \mathrm{s}$ postoperatively $(\mathrm{t}=21.39, \quad P<$ 0.001 ). The preoperative and postoperative neurological functions assessed by NIHSS scores were $1.067 \pm 0.122$ and $1.005 \pm 0.108$, respectively, and this difference was statistically significant $(Z=-3.178, P<0.01)$. Comparison of preoperative and postoperative CTP parameters of the circulating blood supply area (Table 2) showed no significant difference in cerebral blood volume $(\mathrm{CBV}$, $P>0.05)$, whereas there were a significant differences in cerebral blood flow (CBF, increase in CBF postoperatively, $P<0.05$ ), postoperative time to peak (TTP, decrease in TTP postoperatively, $P<0.001$ ), and mean transit time (MTT, decrease in MTT postoperatively, $P<0.01)$.

\section{Results of postoperative complications}

Among the 67 patients, three patients had complications within 30 days after the surgery, including the following: one case $(1.5 \%)$ of complications of a perforating branch event, for which the symptoms of this patient were relieved after active medication treatment for more than a month, whereas mild residual neurological dysfunction remained; one case (1.5\%) of subacute thrombosis development in the stent, which was improved after active intra-arterial thrombolysis, and no residual neurological dysfunction was found (Fig. 2); and one case of

Table 1 Comparison of preoperative and postoperative $\mathrm{TICl}$

\begin{tabular}{lllll}
\hline $\mathrm{TICl}$ & 1 & $2 \mathrm{a}$ & $2 \mathrm{~b}$ & 3 \\
\hline Preoperative & 8 & 47 & 12 & 0 \\
Postoperative & 0 & 0 & 37 & 30 \\
\hline
\end{tabular}

postoperative vs. preoperative, $X^{2}=97.76, P=0.000$ 
Table 2 Comparison of preoperative and postoperative CTP $\left(\mathrm{CBF}, \mathrm{ml} \cdot 100 \mathrm{~g}^{-1} \cdot \mathrm{min}^{-1} ; \mathrm{CBV}, \mathrm{ml} \cdot 100 \mathrm{~g}^{-1} ; \mathrm{TTP}, \mathrm{s} ; \mathrm{MTT}, \mathrm{s}\right)$

\begin{tabular}{lllll}
\hline Time & CBF & CBV & TTP & MTT \\
\hline Preoperative & $52.86 \pm 9.03$ & $30.78 \pm 4.39$ & $13.12 \pm 2.59$ & $7.11 \pm 1.48$ \\
Postoperative & $59.67 \pm 8.14$ & $31.99 \pm 3.71$ & $10.85 \pm 2.31$ & $4.75 \pm 0.81$ \\
T-value & -2.422 & 0.159 & 6.667 & 1.621 \\
P-value & 0.023 & 0.811 & 0 & 0.014 \\
\hline
\end{tabular}

microwire being clamped by the distal vasospasm. This last patient was treated with antivasospasm medication, and the microwire was pulled out the next day after the surgery, after which no residual neurological dysfunction was found. The overall rate of perioperative complications was $4.5 \%(3 / 67)$. No new PCI or TIA occurred in the area receiving blood supply from the offending vessels, and no hyper-perfusion, arterial dissection, or death occurred.

\section{Follow-up results}

Sixty-seven patients who were followed up by telephone, Wechat, or imaging re-examination had an average of $56 \pm 21$ months (36-66 months) of follow-ups, including 55 cases (82\%) that included regular imaging reviews and 12 cases of phone or Wechat follow-ups (the latter 12 patients refused to have imaging-review follow-ups). Four patients (95\% confidence interval [CI] 0.866-0.998) exhibited stenosis in the stent, and three of these four patients had stable symptoms. Continued imaging- review follow-ups showed that one patient had TIA and was consequently treated with balloon dilation. The remaining patients did not have TIA or PCI in the area receiving blood supply from the offending vessels during follow-ups.

\section{Discussion}

ICAS is one of the most common causes of ischemic stroke and has a high risk of recurrence, especially in Asian populations [4, 7]. More than $40 \%$ of ischemic stroke cases are related to impaired blood circulation of the vertebral and basilar arteries. The risk of disease-related ischemic stroke is higher in symptomatic patients with a high-degree of atherosclerotic stenosis $(>70 \%)$ [8]. Patients with ischemic stroke in the posterior circulation usually have more severe symptoms than those with ischemic stroke in the anterior circulation [9]. For ICAS, a number of randomized controlled trials have been conducted to explore the best treatment strategies for preventing the recurrence of ischemic stroke [10-12]. However, the results suggest that intensive drug therapy combined with the control of risk factors has been clearly more efficacious than that of intracranial stenting, which is mainly due to the low recurrence rate of stroke after drug therapy and the relatively high incidence of complications related to endovascular therapy. As such, it may be difficult to perform more efficacious intravascular treatment of ICAS compared to that of
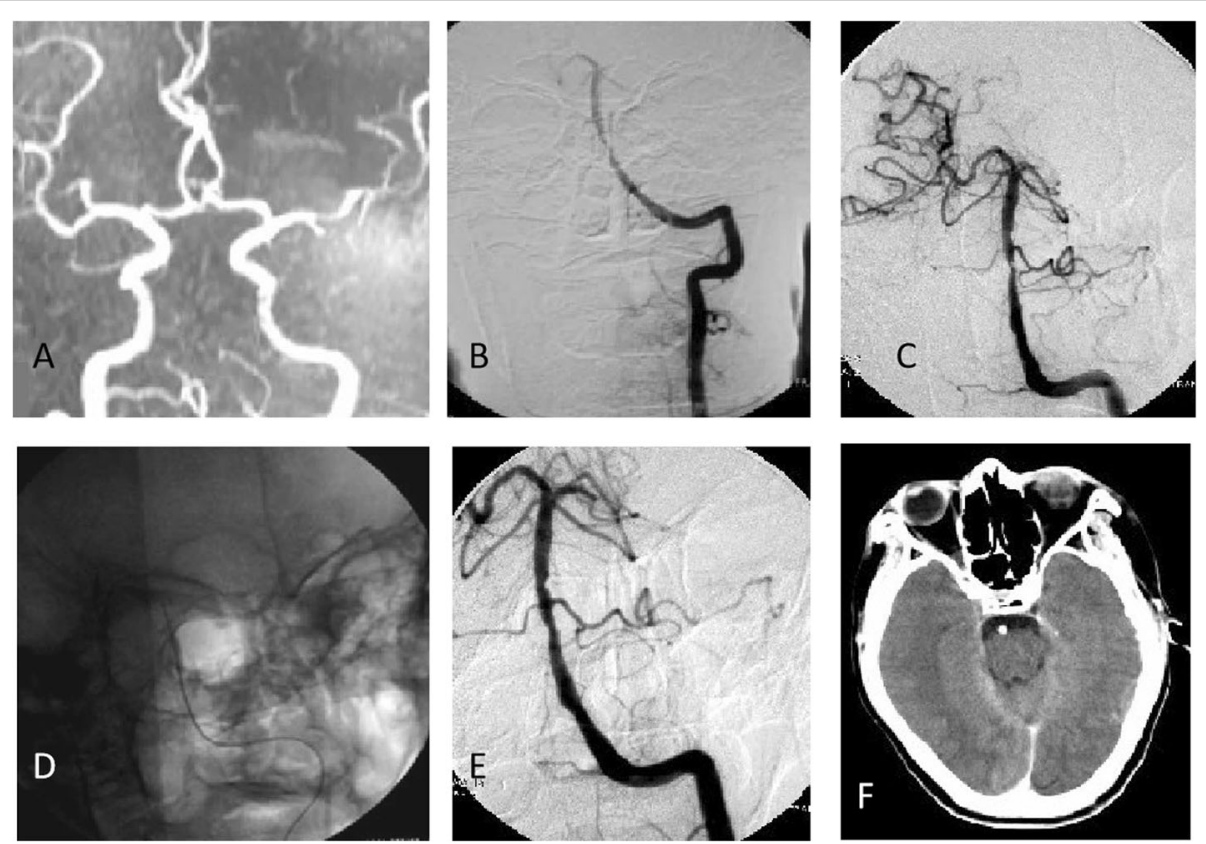

Fig. 2 Management of subacute thrombosis in the stent. a Preoperative MRA images showed subacute occlusion after basilar stenting. b Preoperative DSA images showed acute occlusion after basilar stenting. c-d Intraoperative, intra-arterial thrombolysis and balloon dilation. e Postoperative, subacute occlusion of basilar artery was recanalized. f Postoperative CT images showed no intracranial hemorrhage 
drug therapy. However, according to the SAMMPRIS study, patients who received intensive medication had a $5.8 \%$ primary-endpoint event rate within 30-day follow-ups, $12.6 \%$ one-year endpoint event rate, and a $14.9 \%$ three-year endpoint-event rate. In the VISSIT trial, patients who received intensive medication had an even higher 30-day primary-endpoint event rate and one-year endpoint event rate compared to those reported from the SAMMPRIS study. Although the incidence of endpoint events in patients receiving intensive medication is lower than that of patients who receive intracranial stenting, the incidence of endpoint events is still high and unacceptable. The adverse events in these studies occurred after Winspan stenting, suggesting that such adverse events may be related to the open-loop design, large radial force, high head-end hardness, and severe tortuosity of Wingspan stents that make them difficult to pass through blood vessels; in addition, the Wingspan stent is difficult to operate and can easily induce perforating branch events [13]. Therefore, based on our experience in the treatment intracranial aneurysms, we chose Enterprise stents in our present study, which is a more mature stent for ICAS treatment. In addition, after the above two major studies, the CASSISS trial held in China released the first-stage results in 2015 [14]. The CASSISS trial continuously included 100 patients and implanted Winspan stents in 13 large centers, which showed a success rate of stent implantation of $100 \%$ and a $2 \%$ incidence of stroke and death in the postoperative 30 days, which was significantly lower than the 14.7 and $24.1 \%$ in the SAMMPRIS and the VISSIT trials, respectively. These results provide hope for further exploring ICAS endovascular treatments.

The Enterprise stent is a closed-loop self-expanding stent for assisting spring-coil embolization of widenecked intracranial aneurysms, with a stent diameter of $4.5 \mathrm{~mm}$ and which consists of four different lengths -14 , 22,28 , and $37 \mathrm{~mm}$-that are suitable for $2.5-4.0-\mathrm{mm}$ intracranial blood vessels. The Enterprise stent has a release of less than $70 \%$ and is recyclable. It has a closedloop design so that it is easily passed through curved blood vessels, and its radial support force is smaller than that of the Winspan stent. The operation and release of the Enterprise stent are simpler than those of the Winspan stent, and its positioning is comparatively more accurate. Moreover, the Winspan stent is more prone to cause intimal hyperplasia and irritation to the vessel wall. Not many Enterprise stenting reports for ICAS treatment are currently available. A study by Vajda et al. [15] used the Enterprise stent for the treatment of symptomatic ICAS, with a success rate of $100 \%$. The main complications occurred in four cases $(9.1 \%)$ during the perioperative period (30 days), including three cases
(6.8\%) of ischemic perforating stroke and one case (2.2\%) of reperfusion stroke. Among the 42 patients with a median follow-up of 25.6 months (range of 12-57 months), no further TIA or stroke was found. Among the 38 patients who underwent imaging-review followups, three cases $(6.81 \%)$ experienced $>50 \%$ restenosis in the stent after an average follow-up of 22 months. Our present study showed that for patients with symptomatic ICAs, applications of small-sized balloon angioplasty and the Enterprise stent implantation were technically successful with a lower incidence of complications. A study by Feng et al. [16] used the Enterprise stent for symptomatic ICAS treatment, which yielded a successful operation rate of $100 \%$ and the stenosis decreased from $79.3 \pm 8.1 \%$ to $14.9 \pm 12.3 \%$. The perioperative ischemic stroke rate was $6.8 \%$, and the hemorrhagic stroke rate was $2.2 \%$. There were no further TIA or strokes during an average follow-up of 25.6 months, and restenosis in the stent was $>50 \%$ in $6.8 \%$ of the cases. The promising results of these two studies offer hope for improving ICAS treatments. However, these two studies performed ICAS stent implantations in the anterior circulation. Although they had high successful operation rates and the incidences of short-term and long-term postoperative complications were lower than those in the SAMMPRIS trial, they did not perform detailed subgroup analysis, and the number of cases in the basilar artery group was relatively small. In addition, the specific perioperative complications, long-term results, and restenosis of the basilar artery subgroup were unclear. To further evaluate the long-term effect of Enterprise stents in the treatment of severe basilar artery stenosis, our present study performed long-term follow-ups and ultimately yielded more efficacious results. Two cases were found to have complications within the 30-day postoperative followups, and one case (2.7\%) had complications from perforating branch events. After active treatment, the symptoms of this patient were relieved for more than a month, and mild residual neurological dysfunction was found. Another case (2.7\%) with postoperative complications developed subacute thrombosis in the stent, which was improved after active intra-arterial thrombolysis. Subsequently, no neurological dysfunction remained, with an overall perioperative rate of $5.4 \%(2 / 37)$. No new PCI or TIA occurred in the area receiving blood supply from the offending vessels during follow-ups, and no hyper-perfusion, arterial dissection, or death occurred. For patients who were considered to only have neurological dysfunction as a complication, their overall perioperative complications were $2.7 \%$. The mean follow-up period was $56 \pm 21$ months (36-66 months). Intra-stent stenosis occurred in two patients (5.4\%), and one of them had stable symptoms and continued imagingreview follow-ups. This patient had subacute thrombosis. 
One patient had TIA and underwent balloon dilation. The remaining patient had no TIA or PCI in the area receiving blood supply from the offending vessels during follow-up and they had $2.7 \%$ actual symptomatic stenosis. Collectively, the results of these studies were more promising than those of the restenosis rates of the Wingspan stents (31.2\%).

In 2019, the long-awaited WEAVE trial was published [17], which confirmed the perioperative safety of the Wingspan stent. In this trial, the perioperative stroke and mortality rate was $2.6 \%$, which was better than the set target of a $4 \%$ event incidence. However, the longterm results of the WEAVE trial require further followup. Comparing our results with the WEAVE trial showed that although perioperative complications occurred in two of our cases, basilar artery stenosis accounted for $14 \%$ (22 cases) of cases in the WEAVE trial, including two non-fatal strokes, two deaths from stroke, and a total of four patients who had an index event of stroke, hemorrhage, or death. Of the two nonfatal stroke cases, one patient had stroke in the pontine perforating branch of the basilar artery, with the postoperative modified Rankin Scale (mRS) being reduced to a score of 4. Although the incidence of stroke due to perforator occlusion was only $0.7 \%(1 / 152)$ and the total number of perforator events was low, the events affecting the perforating branches of the basilar artery in the WEAVE trial likely reached $4.5 \%(1 / 22)$, which was higher than that of our present findings. Thus, we conclude that Enterprise stenting is more beneficial in the treatment of basilar artery stenosis compared to that of Wingspan stenting. Further follow-ups of the WEAVE trial are needed because this study did not analyze the long-term efficacy of stenting and restenosis.

Our present study focused on preoperative assessment, perioperative management, surgical timing, surgical experience, perforator protection, and problems of restenosis. We used multimodal imaging to evaluate the lesion length, degree of stenosis, cerebral perfusion, and collateral circulation. Additionally, our multimodal imaging was supplemented with HR-MRI to monitor plaque location, shape, signal, thickness, and presence of localized or circular plagues. We also measured the nearby parenchymal signal as a reference to determine the plague texture, and the plague signals were categorized into equal signal, slightly lower/low signals, slightly higher/high signals, and mixed signals. HR-MRI has also been used to analyze plaque textures and locations to minimize events affecting the perforating branches of the basilar artery, especially for patients with hyperlipidemia and hyperglycemia [18]. In addition, multimodal imaging assessment has also been shown to be beneficial for preventing hyper-perfusion [19]. In the present study, during the perioperative period, we controlled blood pressure at a relatively low level of $120-130 \mathrm{mmHg}$, and we used the implementation of appropriate sedation to reduce the occurrence of cerebral hemorrhage and hyper-perfusion [20]. The bowstring effect should be avoided when loosening the balloon and stent system intraoperatively to prevent tearing of the perforating vessels and to achieve sub-satisfactory results once the balloon is expanded [21]. Perforator stroke (PS) is one of the most common complications of intracranial angioplasty and/or stent implantation [13]. The basilar artery is the most common site of PS among all perforator-rich areas. In the SAMMPRIS trial, the incidence of PS in the basilar artery was $16 \%$. The high perioperative PS rate and its unique blood supply through perforating branches make the basilar artery a key location for studying such complications. Diabetes was associated with a significantly higher incidence of PS $(11.4 \%$ vs. $2.3 \%, P=0.004)$. Dyslipidemia was another risk factor for atherosclerosis and is associated with a high incidence of PS $(7.6 \%$ vs. $1.8 \%$, $P=0.045)$. The incidence of PS in patients with $<18$ days difference between the time of the last symptom to the time of operation was higher than that of the patients with $>18$ days difference $(8.9 \%$ vs. $1.5 \%, P=0.009)$, and $5.1 \%$ of patients were found to be associated with the PS related to surgery. Therefore, we adjusted our surgical timing to be 3 weeks after the last symptom onset [22]. The main cause of restenosis is the development of intimal hyperplasia, which might be related to dilated trauma, stent thrombosis, inflammatory responses of the blood vessel wall to the stent, and high radial force stimulation. The prevention of restenosis in our present study was likely due to reducing the number of balloon expansions, performance of sub-satisfactory expansion, and our choice of the Enterprise stent. Although the Enterprise stent has the capacity of recovery and release, evert effort was made to accurately localize the stent and to complete the release in one attempt. Moreover, the characteristics of the Enterprise stent also reduced the stimulation and cutting of the plague.

\section{Conclusions}

In summary, patients who underwent endovascular treatments were evaluated via multimodal imaging in the present study. Intravascular balloon dilation and angioplasty combined with Enterprise stent implantation was found to be a safe and effective technique for treating severe atherosclerotic stenosis of the basilar artery, which yielded a high success rate, low perioperative complication rate, and achieved efficacious medium- and long-term therapeutic outcomes.

Abbreviations

CICAS: Chinese intracranial atherosclerosis study; ICAS: Intracranial atherosclerotic stenosis; WASID: Warfarin-aspirin symptomatic intracranial disease trial; PCI: Posterior circulation infarct; TIA: Transient ischemic attack; 
HR-MRI: High-resolution magnetic resonance imaging; SAMMPRIS: Stenting versus aggressive medical therapy for intracranial arterial stenosis; CASS ISS: China angioplasty and stenting for symptomatic intracranial severe stenosis; VISSIT: The vitesse stent ischemic therapy; WEAVE: Wingspan stent system post market surveillance

\section{Acknowledgements}

We thank all patients, caregivers, health care professionals, students and institutions who have contributed and collaborated in this study.

\section{Authors' contributions}

Conception and design: YL, LS, WXand ZL. Analysis and interpretation: XG, $\mathrm{DH}$, and XZ. Drafting the manuscript and checking for important intellectual content: YL and ZL. All authors critically read and revised the manuscript, and approved its submission for publication.

\section{Funding}

This study was funded by hunan provincial health and family planning Commission((Grant number C2015-7). The sponsor had no role in the design or conduct of research.

\section{Availability of data and materials}

The datasets used and/or analyzed during the current study are available from the corresponding author on reasonable request.

\section{Ethics approval and consent to participate}

The study is conducted in accordance with the Declaration of Helsinki. Potential participants receive detailed written and oral information on the study procedures and all participants provide written informed consent. Ethics approval of the study was obtained from the Hengyang Central Hospital and Southern Medical University.

\section{Consent for publication}

Not applicable.

\section{Competing interests}

The authors declare that they have no competing interests.

\section{Author details}

'Department of Neurosurgery, Affiliated Hengyang Hospital, Southern Medical University (Hengyang Central Hospital), Hengyang 421001, China. ${ }^{2}$ Department of Vascular Surgery, Affiliated Hengyang Hospital, Southern Medical University (Hengyang Central Hospital), 12\# Yancheng road, Hengyang 421001, Hunan province, China. ${ }^{3}$ Department of Neurointervention, Affiliated Tiantan Hospital, Capital Medical University, 119\# west south fourth ring road, fengtai district, Beijing 100050, China. ${ }^{4}$ Department of Gynecology, Affiliated Hengyang Hospital, Southern Medical University (Hengyang Central Hospital), Hengyang 421001, China. ${ }^{5}$ Department of Science and Education, Affiliated Hengyang Hospital, Southern Medical University (Hengyang Central Hospital), Hengyang 421001, China. ${ }^{6}$ Department of Nutrition, Affiliated Hengyang Hospital, Southern Medical University (Hengyang Central Hospital), Hengyang 421001, China. ${ }^{7}$ Department of Neurosurgery, Zhujiang Hospital, Southern Medical University, Guangzhou 510282, China.

Received: 27 April 2020 Accepted: 13 July 2020

Published online: 31 July 2020

\section{References}

1. Wang W, Jiang B, Sun $H$, et al. Prevalence, incidence, and mortality of stroke in China. Circulation. 2017;135:759-71.

2. Wang Z, Hu S, Sang S, et al. Age-period-cohort analysis of stroke mortality in China data from the global burden of disease study 2013. Stroke. 2017; 48(2):271-5.

3. Wang J, Zhong CY, Zhang Y, Wei Y, Liu H, Wu C, et al. Seven years' followup of compara -tive study between stenting and medication for treatment of sym -ptomatic vertebrobasilar artery stenosis. Interv Neuroradiol. 2018; 24(1):43-50.

4. Wang Y, Zhao X, Liu L, et al. Prevalence and outcomes of symptomatic intracranial large artery stenoses and occlusions in China: the Chinese intracranial atherosclerosis (CICAS) study. Stroke. 2014;45:663-9.
5. Yu W, Jiang W-J. Stenting for intracranial stenosis: potential future for the prevention of disabling or fatal stroke. Stroke Vasc Neurol. 2018;3(3):140-6.

6. Warfarin-Aspirin Symptomatic Intracranial Disease (WASID) Study Group. Prognosis of patients with symptomatic vertebral or basilar artery stenosis. Stroke. 1998;29(7):1389-92.

7. Qureshi Al, Caplan LR. Intracranial atherosclerosis. Lancet. 2014;383(9921): 984-98.

8. Wang J, Zhong CY, Zhang Y, et al. Seven years' follow-up of com -parative study between stenting and medication for treatment of symptomatic vertebrobasilar artery stenosis. Interv Neuroradiol. 2018;24(1):43-50.

9. Jiang WJ, Xu XT, Du B, Dong KH, Jin M, Wang QH, et al. Long-term outcome of elective stenting for symptomatic intracranial vertebrobasilar stenosis. Neurology. 2007;68:856-8.

10. Derdeyn CP, Chimowitz MI, Lynn MJ, et al. Aggressive medical treatment with or without stenting in high-risk patients with intracranial artery stenosis (SAMMPRIS): the final results of a randomised trial. Lancet. 2014;383: 333-41.

11. Zaidat OO, Fitzsimmons BF, Woodward BK, et al. Effect of a balloonexpandable intracranial stent vs medical therapy on risk of stroke in patients with symptomatic intracranial stenosis: the VISSIT randomized clinical trial. JAMA. 2015;313:1240-8.

12. Lutsep HL, Lynn MJ, Cotsonis GA, et al. Does SAMMPRIS support stenting over medical therapy for subpopulations of patients with intracranial arterial stenosis? Stroke. 2015;46(11):3282-4.

13. Jia B, Liebeskind DS, Ma N, et al. Factors associated with perforator stroke after selective basilar artery angioplasty or stenting. J Neurolntervent Surg. 2017:9:738-42.

14. Gao P, Jiao LQ, Ling F, et al. China angioplasty and stenting for symptomatic intracranial severe stenosis (CASSISS): a new, prospective, multicenter, randomized controlled trial in China. Interv Neuroradiol. 2015. 21(2):196-204.

15. Vajda Z, Schmid E, Lindner A, et al. The modified Bose method for the endovascular treatment of intracranial atherosclerotic arterial stenoses using the Enterprise stent. Neurosurgery. 2012;70(1):91-101.

16. Feng $Z$, Duan $G$, Huang $H$, et al. Enterprise stent for the treatment of symptomatic intracranial atherosclerotic stenosis: an initial experience of 44 patients. BMC Neurol. 2015:15:187.

17. Alexander MJ, Chaloupka JC, Zauner A, et al. WEAVE intracra-nial stent trial: final trial results in 150 patients treated on-label. Stroke. 2019:50:889-94.

18. Nordmeyer H, Chapot R, Aycil A, et al. Angioplasty and stenting of intracranial arterial stenosis in perforator-bearing segments: a comparison between the anterior and the posterior circulation. Front Neurol. 2018:9:533.

19. Chen XQ, Tian JM, Zuo CJ, et al. Hemodynamic alterations in unilateral chronic middle cerebral artery stenosis patients and the effect of percutaneous transluminal angioplasty and stenting: a perfusion-computed tomography study. Acta Radiol. 2015;56(6):754-60

20. Salik AE, Selcuk HH, Zalov H, et al. Medium-term results of undersized angioplasty and stenting for symptomatic high-grade intracranial atherosclerotic stenosis with Enterprise. Interv Neuroradiol. 2019;25(5):484-90.

21. Wang J, Zhong CY, Zhang $Y$, et al. Seven years' follow-up of comparative study between stenting and medication for treatment of symptomatic vertebrobasilar artery stenosis. Interv Neuroradiol. 2018;24(1):43-50.

22. Alexander MD, Rebhun JM, Hetts SW, et al. Lesion location, stability, and pretreatment management: factors affecting outcomes of endovascular treatment for vertebrobasilar atherosclerosis. J Neurointerv Surg. 2016;8:466-70.

\section{Publisher's Note}

Springer Nature remains neutral with regard to jurisdictional claims in published maps and institutional affiliations. 